\title{
Carbonyls as Latent Alkyl Carbanions for Conjugate Additions
}

\author{
Xi-Jie Dai ${ }^{+}$,Haining Wang $^{+}$, and Chao-Jun Li*
}

\begin{abstract}
Conjugate addition of carbon nucleophiles to electron-deficient olefins is one of the most powerful methods for forming carbon-carbon bonds. Despite great achievements in controlling the selectivity, variation of the carbon nucleophiles remains largely underexplored, with this approach relying mostly on organometallic reagents. Herein, we report that naturally abundant carbonyls can act as latent carbon nucleophiles for conjugate additions through a rutheniumcatalyzed process, with water and nitrogen as innocuous byproducts. The key to our success is homogeneous ruthenium(II) catalysis, combined with phosphines as spectator ligands and hydrazine as the reducing agent. This chemistry allows the incorporation of highly functionalized alkyl fragments into a vast array of electron-deficient olefins under mild reaction conditions in a reaction complementary to the classical organometallic-reagent-based conjugate additions mediated or catalyzed by "soft" transition metals.
\end{abstract}

Conjugate addition of carbon nucleophiles to electrondeficient olefins represents one of the most reliable alkylation strategies for carbon-carbon bond formation with exclusive 1,4-regioselectivity. ${ }^{[1]}$ Traditional conjugate additions to $\alpha, \beta$ unsaturated carbonyl compounds and related electron-deficient olefins are generally accomplished in two ways: 1) through a "soft" enolization of carbonyl derivatives bearing acidic methylene protons (Figure $1 \mathrm{~A}),{ }^{[1 \mathrm{a}-\mathrm{d}]}$ and 2 ) through a "soft" transition-metal-mediated or -catalyzed addition process with metals such as copper and ${ }^{[2]}$ rhodium, ${ }^{[3]}$ and among others, ${ }^{[4]}$ whereby stoichiometric organometallic or organometalloid reagents serve as carbon nucleophiles (Figure $1 \mathrm{~B}) .{ }^{[5]}$ Relative to the tremendous progress in controlling the selectivity, especially the stereoselectivity, ${ }^{[2, i, 3,4]}$ the choice of carbon nucleophiles remains limited, in spite of the importance of this issue for chemical diversification. Taking the most important organometallic-reagent-based method as an example, stoichiometric amounts of metal are essential to generate carbon nucleophiles from petroleumderived organohalides. Furthermore, the high reactivity and basicity of most organometallic reagents often make it challenging to realize broad functional-group tolerance and demand strict control in terms of low-temperature, anhy-

[*] X.-J. Dai, ${ }^{[+]}$Dr. H. Wang, ${ }^{[+]}$Prof. Dr. C.-J. Li

Department of Chemistry and FQRNT Centre for

Green Chemistry and Catalysis, McGill University

801 Sherbrooke St. W., Montreal, Quebec H3A OB8 (Canada)

E-mail: cj.li@mcgill.ca

$\left.{ }^{+}\right]$These authors are co-first authors and contributed equally to this work.

(2) Supporting information and the ORCID identification number(s) for

(DD the author(s) of this article can be found under:

https://doi.org/10.1002/anie.201700059.

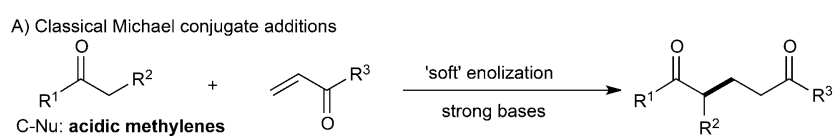

B) Traditional organometallic-reagent-based conjugate additions

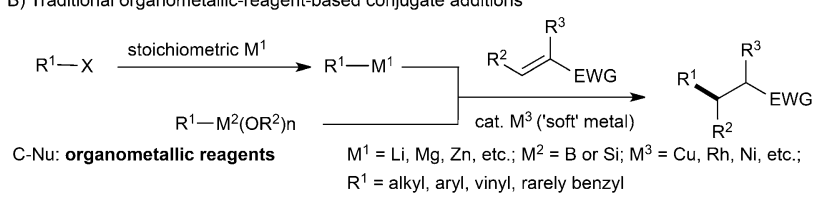

C) Carbonyls as alkyl carbanions for conjugate additions

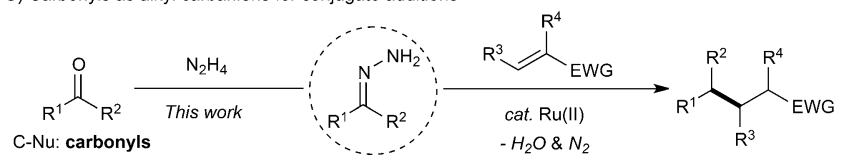

Figure 1. Carbon nucleophiles used in conjugate additions for the formation of new $\mathrm{C}-\mathrm{C}$ bonds. a) Carbonyl derivatives bearing acidic methylene protons are employed as carbon nucleophiles in the classical Michael addition. b) Traditional "soft" metal-mediated or -catalyzed conjugate additions rely on organometallic or organometalloid reagents as carbon nucleophiles. c) Carbonyl compounds were discovered to act as latent alkyl carbanions via hydrazone formation for conjugate additions catalyzed by ruthenium with phosphine ligand (this work). C-Nu= carbon nucleophile; cat. = catalytic, EWG =electron-withdrawing group.

drous, and oxygen-free reaction conditions. ${ }^{[2 \mathrm{~h}]}$ Rare variants of carbon nucleophiles, along with innate constraints imposed by organometallic reagents, prompted us to explore viable carbanion alternatives for conjugate addition reactions. Herein, we report a ruthenium(II)-catalyzed conjugate addition of carbonyl compounds ${ }^{[6]}$ masked as "soft" alkyl carbanions, through polarity reversal, ${ }^{[7]}$ to a wide range of electrondeficient olefins under mild reaction conditions (Figure 1C) in a reaction complementary to the organometallic-reagentbased conjugate additions mediated or catalyzed by "soft" transition metals.

Very recently, we developed a ruthenium-based catalytic system for direct deoxygenation of primary aliphatic alcohols, which proved to be highly chemo- and regio-selective with both simple and complex compounds. ${ }^{[8 a]}$ Capitalizing on the ruthenium complex (A, Figure 2) postulated in the deoxygenation reaction, we made another significant discovery by engaging carbonyl compounds (B, Figure 2) to form new carbon-carbon bonds, possibly via a six-membered ring, chair-like transition state (C, Figure 2).$^{[9 \mathrm{a}]}$ We speculated that the polarized carbon-carbon double bonds in electrondeficient olefins might be an equally reactive substitute for carbonyl compounds in $\mathbf{C}$ (D, Figure 2). In addition, the soft nature of ruthenium(II), which bears a resemblance to "soft" metals in classical conjugate addition, ${ }^{[10]}$ led us to question whether such homogenous ruthenium(II) catalysis could be 


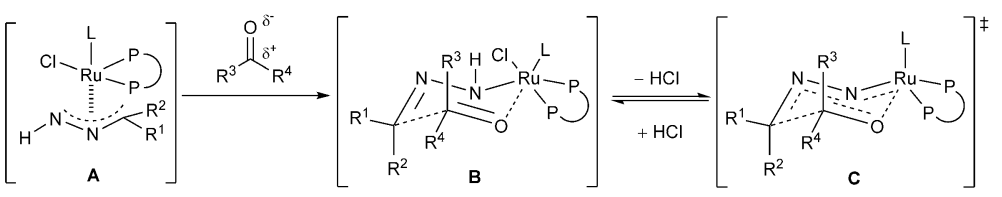

$\mathrm{HCl} \underbrace{\mathrm{R}^{3} \mathrm{\delta}^{4}}_{\delta^{+}} \mathrm{EWG}$
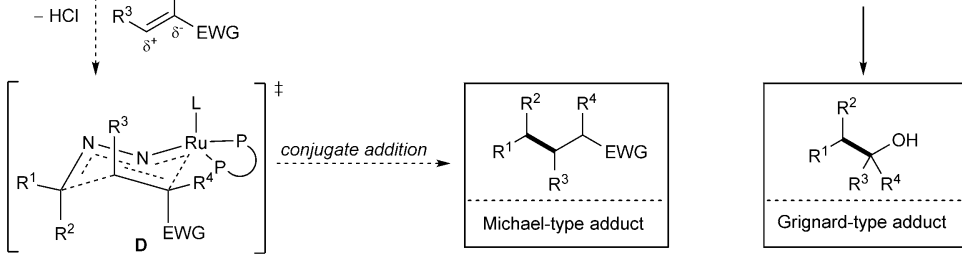

Figure 2. Mechanistic hypothesis of using carbonyls as alkyl carbanions for conjugate additions. Inspired by the postulated ruthenium complex $\mathbf{A}$ in deoxygenation chemistry, and its recent proof-of-concept application for $\mathrm{C}-\mathrm{C}$ bond formation through carbonyl addition, we hypothesized that $\mathbf{A}$ could be intercepted by Michael acceptors to produce conjugate addition products. TS $=$ transition state.

even more effective for conducting conjugate additions than carbonyl additions. To verify this hypothesis, benzaldehyde $\mathbf{1 a}$ and tert-butyl acrylate $\mathbf{2} \mathbf{a}$ were chosen as model substrates. The preformed hydrazone from $\mathbf{1}$ a was treated with $\mathbf{2} \mathbf{a}$ in the presence of $\left[\mathrm{Ru}(p \text {-cymene }) \mathrm{Cl}_{2}\right]_{2}, 1,2$-bis(dimethylphosphino)ethane (dmpe, $\mathbf{L}_{3}$ ), and $\mathrm{K}_{3} \mathrm{PO}_{4}$ in THF solution. To our delight, the desired Michael-type 1,4adduct 3 a was obtained in $76 \%$ yield at $50{ }^{\circ} \mathrm{C}$ after $5 \mathrm{~h}$, with a stoichiometric amount of $\mathrm{CsF}$ as an additive (Scheme 1, entry 3 ). ${ }^{[11]}$ It should be noted that no desired 1,4-adduct was produced in the absence of the ruthenium(II) precatalyst, ${ }^{[12]}$ and a significantly lower yield was obtained without the participation of either phosphine ligands or cesium fluoride $(40 \%$ and $65 \%$ yield as measured by ${ }^{1} \mathrm{H}$ NMR, respectively). Our early investigation into spectator ligands bound to the ruthenium(II) precatalyst suggested that significant enhancement in catalyst activity is achieved when using electron-rich phosphine ligands. ${ }^{[\mathrm{a}, 9]} \mathrm{In}$ contrast, strong $\sigma$ donors other than phosphines, including N-heterocyclic carbenes (NHCs) and charge-neutral amido ligands, were largely inferior. Aligned with this observation, studies on the influence of various electron-rich phosphine ligands were prioritized for optimization (Scheme 1). In fact, the conjugate addition of benzaldehyde-derived hydrazone to $\mathbf{2 a}$ proceeded smoothly as long as certain phosphines were used as dative ligands, regardless of their denticity. Nevertheless, varying degrees of catalyst activity were observed, resulting in yield variations for 3a. For instance, monodentate tricyclohexylphosphine $\left(\mathrm{PCy}_{3}, \mathbf{L}_{2}\right)$ was less efficient than bidentate 1,4-bis(dicyclohexylphosphino)butane (dcpb, $\mathbf{L}_{9}$; entry 2 vs. 9). However, the use of trimethylphosphine $\left(\mathrm{PMe}_{3}, \mathbf{L}_{\mathbf{1}}\right)$ and $\mathbf{L}_{\mathbf{3}}$ afforded comparable yields (entry 1 vs. 3 ), presumably owing to their similar electronic and steric nature. On the other hand, diphenylphosstandard. phines linked by alkylidene bridges outperformed those with other linkers, including 1,1'-bis(diphenylphosphino)ferrocene (dppf, $\mathbf{L}_{\mathbf{1 0}}$ ), 2,2'-bis(diphenylphosphino)-1,1'-binaphthyl (BINAP, $\mathbf{L}_{\mathbf{1 1}}$ ), and 4,5-bis(diphenylphosphino)-9,9-dimethylxanthene (Xantphos, $\mathbf{L}_{\mathbf{1 2}}$; entries 5-8 vs. 10-12). In the former case, an alkylidene linker with three carbons is optimal, since attenuated reactivity was shown in others with either longer or shorter linkers. Finally, we concluded that 1,3-bis(diphenylphosphino)propane (dppp, $\mathbf{L}_{7}$ ) was the optimal spectator ligand for binding to $[\mathrm{Ru}(p$-cymene) $\left.\mathrm{Cl}_{2}\right]_{2}$ in the current reaction.

Under the optimized reaction conditions, 3a was obtained in $91 \%$ yield (Scheme 1, entry 7). The scope of the optimized conjugate addition process was initially explored using aromatic carbonyl compounds as carbon nucleophiles in the presence of $\mathbf{L}_{7}$. In general, moderate to excellent yields were obtained using a broad range of electron-rich and electron-poor aromatic aldehydes (Scheme 2, 3a-n). A number of functional groups, including an allyl phenol ether $(\mathbf{3 h})$, aryl ethers $(\mathbf{3 g}, \mathbf{3 i})$, aryl halides $(\mathbf{3 b}, \mathbf{3 c})$, and trifluoromethyls $(\mathbf{3 d}, \mathbf{3 e})$ were compatible with

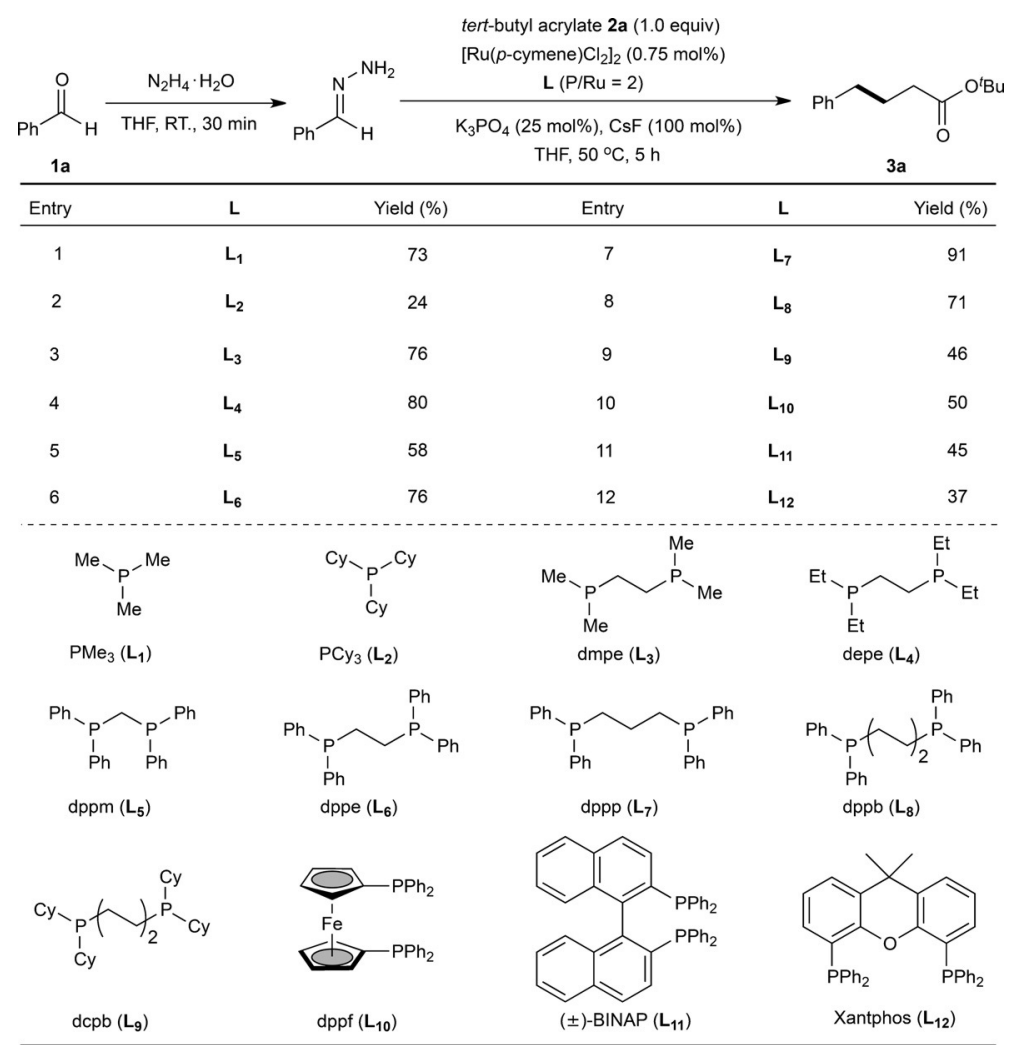

Scheme 1. Effect of various phosphine ligands. Reaction conditions: 1 a $(25 \mu \mathrm{L}$, $0.24 \mathrm{mmol}), \mathrm{N}_{2} \mathrm{H}_{4} \cdot \mathrm{H}_{2} \mathrm{O}(13 \mu \mathrm{L}, 0.26 \mathrm{mmol})$, THF (100 $\left.\mu \mathrm{L}\right)$, room temperature, $30 \mathrm{~min} ; 2 \mathrm{a}(30 \mu \mathrm{L}, 0.2 \mathrm{mmol})$, [Ru(p-cymene) $\left.\mathrm{Cl}_{2}\right]_{2}(0.9 \mathrm{mg}, 0.75 \mathrm{~mol} \%)$, $\mathrm{L}_{1}$ and $\mathrm{L}_{2}$ (3.0 mol\%), or $\mathrm{L}_{3}-\mathrm{L}_{12}$ (1.5 mol\%), $\mathrm{K}_{3} \mathrm{PO}_{4}$ (10.6 mg, $\left.25 \mathrm{~mol} \%\right), \mathrm{CsF}$ (30 mg, $100 \mathrm{~mol} \%$ ), $50^{\circ} \mathrm{C}, 5 \mathrm{~h}$, under $\mathrm{N}_{2}$. The volume of $\mathrm{N}_{2} \mathrm{H}_{4} \cdot \mathrm{H}_{2} \mathrm{O}$ was measured more precisely using the prepared stock THF solution (details in the Supporting Information). Yields were determined by ${ }^{1} \mathrm{H}$ NMR using mesitylene as an internal 


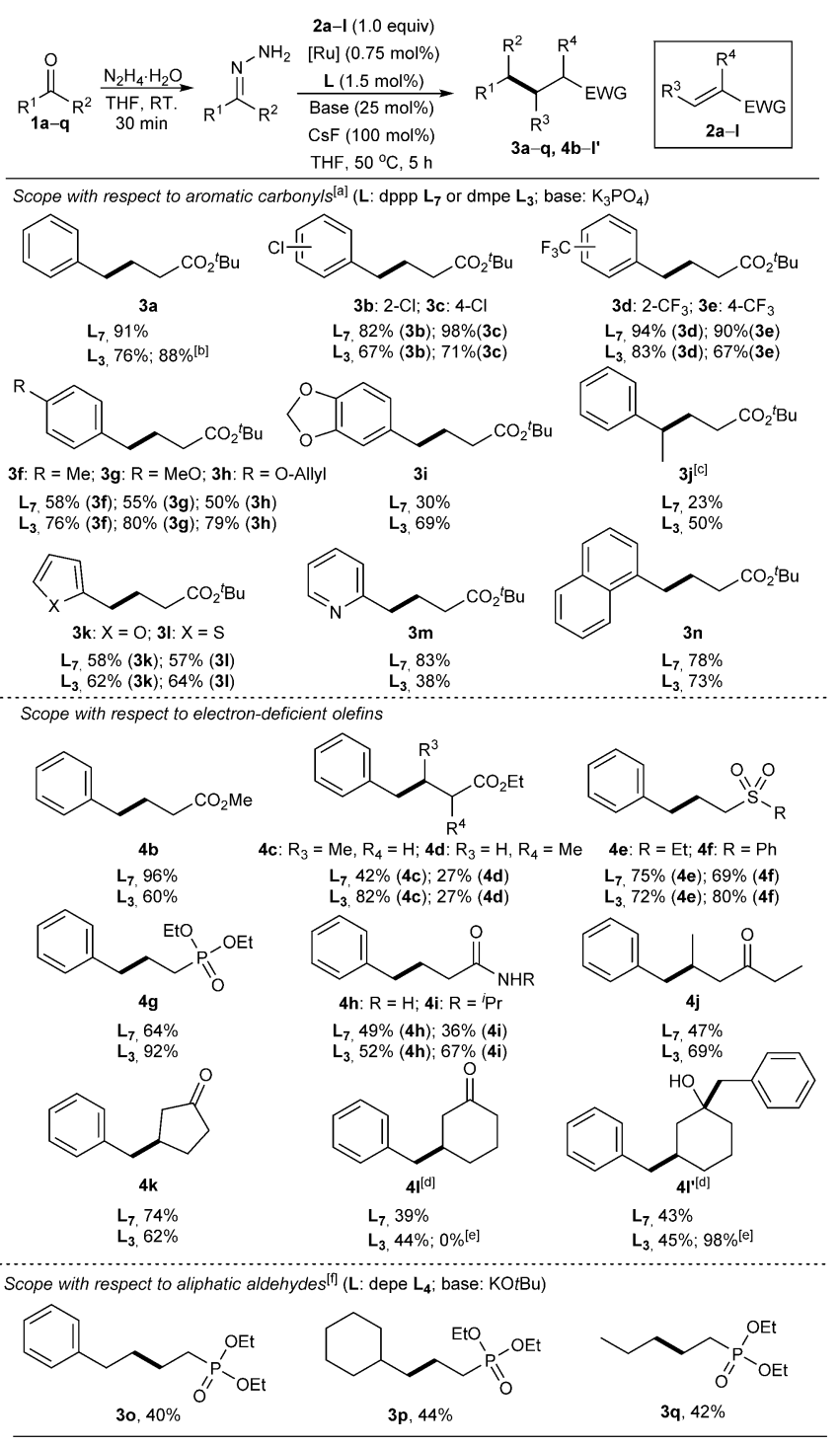

Scheme 2. Scope of conjugate additions with carbonyls as masked alkyl carbanions ( $\mathbf{3} \mathrm{a}-\mathrm{q}$ : from $\mathbf{1} \mathrm{a}-\mathrm{q}$ reacting with $\mathbf{2} \mathrm{a}$ or $\mathbf{2 g} ; \mathbf{4 b -} \mathrm{I}^{\prime}$ : from 1 a reacting with $\mathbf{2} \mathrm{b}-\mathrm{I})$. [a] Reaction conditions: $1 \mathrm{a}-\mathrm{I}(0.24 \mathrm{mmol})$, $\mathrm{N}_{2} \mathrm{H}_{4} \cdot \mathrm{H}_{2} \mathrm{O}(13 \mu \mathrm{L}, 0.26 \mathrm{mmol})$, THF $(100 \mu \mathrm{L})$, room temperature, $30 \mathrm{~min} ; 2 \mathrm{a}-\mathrm{I}(0.2 \mathrm{mmol}),\left[\mathrm{Ru}(p \text {-cymene }) \mathrm{Cl}_{2}\right]_{2}$ (0.9 mg, $\left.0.75 \mathrm{~mol} \%\right)$, L: $\mathrm{L}_{7}$ or $\mathrm{L}_{3}(1.5 \mathrm{~mol} \%), \mathrm{K}_{3} \mathrm{PO}_{4}(10.6 \mathrm{mg}, 25 \mathrm{~mol} \%), \mathrm{CsF}(30 \mathrm{mg}$, $100 \mathrm{~mol} \%), 50^{\circ} \mathrm{C}, 5 \mathrm{~h}$, under $\mathrm{N}_{2}$. Isolate yields were reported. [b] Gram-scale synthesis was conducted (2a, $10 \mathrm{mmol}$ ). [c] Hydrazone synthesis and the subsequent conjugate addition reaction were conducted at $80^{\circ} \mathrm{C}$ for $24 \mathrm{~h}$. $\mathrm{L}_{3}(0.5 \mu \mathrm{L}, 1.5 \mathrm{~mol} \%)$, KOt-Bu $(5.6 \mathrm{mg}$, $25 \mathrm{~mol} \%$ ). [d] $4 \mathrm{I}$ and $4 \mathrm{I}^{\prime}$ were isolated as a mixture in the reaction. [e] Hydrazone (2.4 equiv) was prepared from $1 \mathrm{a}(0.48 \mathrm{mmol})$ and $\mathrm{N}_{2} \mathrm{H}_{4} \cdot \mathrm{H}_{2} \mathrm{O}$ (26 $\left.\mu \mathrm{L}, 0.52 \mathrm{mmol}\right)$. [f] 1 o--q (0.24 mmol), $2 \mathrm{~g}(0.2 \mathrm{mmol})$, L: $\mathrm{L}_{4}(0.7 \mu \mathrm{L}, 1.5 \mathrm{~mol} \%)$, Base: KOt-Bu (5.6 mg, $\left.25 \mathrm{~mol} \%\right)$. The volume of $\mathrm{L}_{4}$ was measured more precisely using the prepared stock THF solution.

this transformation. Heteroaromatic aldehydes containing furans $(\mathbf{1 k})$, thiophenes (1) , and pyridines $(\mathbf{1} \mathbf{m})$ were also effective as nucleophilic coupling partners. A formally similar conjugate addition reaction (analogous to the formation of $\mathbf{3 k}$ from $1 \mathbf{k}$ ) could be carried out through a step-wise Kishner reduction of 2-furylhydrazone and the ene reaction. ${ }^{[13]}$
Surprisingly, 2-pyridyl substituent does not cause any attenuation in catalyst reactivity, despite the fact that it is a wellknown chelating ligand in transition metal catalysis. ${ }^{[14]}$ On the contrary, steric hindrance proves to be a stronger factor in catalytic reactivity, since significantly lower yield was obtained with aromatic ketone $\mathbf{1} \mathbf{j}$, even at elevated temperature, compared to aldehyde counterpart 1a. In addition, poor to moderate yields were observed for electron-rich aromatic aldehydes $(\mathbf{3} \mathbf{f}-\mathbf{i})$. To improve catalyst activity, a more cost-effective bidentate alkylphosphine (dmpe, $\mathbf{L}_{\mathbf{3}}$ : a stronger $\sigma$ donor but a weaker $\pi$ acceptor than $\mathbf{L}_{7}$ ) was chosen instead of 1,2-bis(diethylphosphino)ethane (depe, $\mathbf{L}_{\mathbf{4}}$ ), which was better in the model study (Scheme 1, entry 4 vs. 3 ). Indeed, the switch from $\mathbf{L}_{7}$ to $\mathbf{L}_{3}$ led to higher conversions and yields across all electron-rich aromatic aldehydes $\left(\mathbf{3} \mathbf{f}-\mathbf{i}, \mathbf{L}_{\mathbf{3}}\right.$ vs. $\left.\mathbf{L}_{7}\right)$. Notably, this ligand switch overcame the steric disadvantage of aromatic ketones, providing a modest yield improvement ( $\mathbf{3} \mathbf{j}, \mathbf{L}_{\mathbf{3}}$ vs. $\mathbf{L}_{7}$ ). In contrast to the yield increase for electron-rich aromatic aldehydes, a decrease in yield was detected for most electron-deficient counterparts (1a-e, $1 \mathbf{~ m})$. Nevertheless, a synthetically valuable feature of the current reaction is its ability to incorporate highly functionalized benzyl groups into $\alpha, \beta$-unsaturated esters through conjugate addition. ${ }^{[15]}$ Such benzyl incorporation has long been a nontrivial challenge in the classical organometallic-reagent-based methods. Importantly, an effective gram-scale synthesis of $\mathbf{3 a}$ $(1.94 \mathrm{~g}, 88 \%)$ was carried out to demonstrate the practicability of the current method (Scheme 2, 3a with $\mathbf{L}_{\mathbf{3}}$ ).

Next, the scope with respect to the electron-deficient olefins was surveyed. Under standard reaction conditions, a broad spectrum of electron-deficient olefins were successfully coupled with benzaldehyde-derived hydrazone to give the corresponding 1,4-addition products in moderate to excellent yields (Scheme 2, 4b-l). Specifically, esters (4a-d), ketones $(\mathbf{4} \mathbf{j}-\mathbf{l})$, sulfones $(\mathbf{4 e}, \mathbf{4} \mathbf{f})$, phosphonates $(\mathbf{4} \mathbf{g})$, and amides $(\mathbf{4 h}, \mathbf{4 i})$ were all accommodated, thus demonstrating the mildness of the reaction conditions and the broad functional-group tolerance of this method. Exclusive 1,4regioselectivity was observed for the acyclic enone and 2cyclopentenone $(\mathbf{4 j}, \mathbf{4 k})$. In the case of 2-cyclohexenone, however, cyclic tertiary alcohol $\mathbf{4 I}^{\prime}$ was generated through dibenzylation of $\mathbf{2} \mathbf{l}$ in a slightly higher yield than the desired monobenzylated product $4 \mathbf{l}\left(\mathbf{L}_{7}, \mathbf{4 \mathbf { I } ^ { \prime }}\right.$ vs. $\left.\mathbf{4 I}\right)$. Doubling the amount of hydrazone (2.4 equiv) prepared from $\mathbf{1 a}$ led to the exclusive formation of $\mathbf{4} \mathbf{I}^{\prime}$ in $98 \%$ yield. Intriguingly, the unorthodox dibenzylation featuring successive 1,4- and 1,2addition did not occur with $\mathbf{2 k}$. This striking reactivity difference between $\mathbf{2} \mathbf{k}$ and $\mathbf{2 1}$ likely stems from the torsional ring-strain increase in 5-membered rings, since the $\mathrm{sp}^{2}$ hybridized carbon atom could have changed to an $\mathrm{sp}^{3}$ hybridized carbon atom through benzylation. Consistent with the negative steric influence seen earlier in aromatic ketones, the reactivity of sterically bulky olefins dropped dramatically. For example, compared to the linear propionates $\mathbf{2} \mathbf{a}$ and $\mathbf{2} \mathbf{b}$, a methyl substituent at the $\alpha$ position of propionate $\mathbf{2} \mathbf{d}$ caused a drastic reduction in yield. By contrast, minor steric influence on reactivity was noticed in $\beta$-branched propionate $\mathbf{2 c}$. In cases where the use of $\mathbf{L}_{7}$ provided low yields, a ligand switch to $\mathbf{L}_{\mathbf{3}}$ was generally necessary to 
increase the yields $(\mathbf{4 b}, \mathbf{4} \mathbf{f}-\mathbf{j})$, albeit with a few exceptions (4d and $\mathbf{4 1}$ ).

To further exploit the versatility of this method, aliphatic aldehydes bearing different substituents (arylmethyl, cyclohexyl, and ethyl) were examined in conjugate additions to diethyl vinylphosphonate $\mathbf{2 g}$ (Scheme 2, 3o-q). Unfortunately, performing these reactions under standard conditions only afforded trace amounts of the corresponding 1,4addition products. Enlightened by our previous study on carbonyl and imine additions, ${ }^{[9]}$ we found two critical factors that enhance the reactivity of aliphatic aldehydes: basicity and choice of ligand. Combination of the stronger base $\mathrm{KO} t$ $\mathrm{Bu}$ and the more potent phosphine ligand $\mathbf{L}_{\mathbf{4}}$ delivered modest yields of the desired alkyl phosphonates in all cases. Although preliminary, success in coupling aliphatic aldehydes with electron-deficient olefins through conjugate addition is exciting because the majority of natural carbonyl compounds belong to this class.

In summary, we have developed carbonyls as latent alkyl carbanions for conjugate additions through ruthenium(II)catalyzed reductive coupling, with hydrazine as the key reductant. Such carbon nucleophiles can react with various electron-deficient olefins in a manner that is complementary to the "soft" metal-based carbanions in the classical conjugate additions. This reaction proceeds under mild conditions and tolerates a variety of functional groups on both coupling partners. Efforts to elucidate the mechanism, expand the range nucleophilic carbonyl partners, and develop an asymmetric variant are ongoing in our laboratory.

\section{Experimental Section}

Representative procedure (gram-scale synthesis): A flame-dried flask $\left(50 \mathrm{~cm}^{3}\right)$ equipped with a magnetic stir bar was charged with $\left[\mathrm{Ru}(p \text {-cymene }) \mathrm{Cl}_{2}\right]_{2}(46 \mathrm{mg}, 0.075 \mathrm{mmol}, 0.75 \mathrm{~mol} \%)$ and $\mathrm{K}_{3} \mathrm{PO}_{4}$ $(0.53 \mathrm{~g}, 2.5 \mathrm{mmol}, 25 \mathrm{~mol} \%)$. The flask was transferred into the glove box and charged with dmpe ( $25 \mu \mathrm{L}, 0.15 \mathrm{mmol}, 1.5 \mathrm{~mol} \%)$ and CsF (1.52 g, $10 \mathrm{mmol}, 100 \mathrm{~mol} \%)$ before being sealed with a rubber septum. The flask was then moved out of the glove box and sequentially charged with tert-butyl acrylate $(\mathbf{2} \mathbf{a} ; 1.46 \mathrm{~mL}, 10 \mathrm{mmol}$, 1.0 equiv) and "hydrazone solution" (ca. $6.8 \mathrm{~mL}$, see below) under $\mathrm{N}_{2}$ atmosphere. The reaction mixture was then heated to $50^{\circ} \mathrm{C}$ in an oil bath. Upon stirring for $5 \mathrm{~h}$, the reaction mixture was filtered through a plug of silica gel with EtOAc $(50 \mathrm{~mL})$ as the eluent, concentrated, and purified by flash chromatography (hexane/ethyl acetate 90:10 as the eluent) to give the corresponding product $\mathbf{3} \mathbf{a}$ as a colorless oil (1.94 g, $88 \%$ yield). Hydrazone solution: A mixture of benzaldehyde (1a) $1.22 \mathrm{~mL}, 12 \mathrm{mmol}, 1.2$ equiv) and hydrazine monohydrate ( $630 \mu \mathrm{L}, 13 \mathrm{mmol}, 64-65 \mathrm{wt} \%, 1.3$ equiv) in THF $(5 \mathrm{~mL})$ was stirred at room temperature for $30 \mathrm{~min}$. Prior to injection of this hydrazone solution into the reaction mixture, a small amount of anhydrous $\mathrm{Na}_{2} \mathrm{SO}_{4}$ was added.

\section{Acknowledgements}

The authors acknowledge the Canada Research Chair Foundation (to C.-J.L.), the CFI, FQRNT Center for Green Chemistry and Catalysis, NSERC, and McGill University for financial support. X.-J.D. thanks the chemistry department for the Pall Dissertation Award.

\section{Conflict of interest}

The authors declare no conflict of interest.

Keywords: carbonyls · conjugate addition .

homogeneous catalysis · hydrazine $\cdot$ ruthenium

How to cite: Angew. Chem. Int. Ed. 2017, 56, 6302-6306

Angew. Chem. 2017, 129, 6399-6403

[1] Selected books or chapters on the $\mathrm{C}-\mathrm{C}$ bond formation in organic synthesis via conjugate addition reactions: a) P. Perlmutter, in Conjugate Addition Reactions in Organic Synthesis, Elsevier, Amsterdam, 2013; b) H.-G. Schmalz, in Comprehensive Organic Synthesis, Vol. 4 (Eds.: B. M. Trost, I. Fleming), Pergamon, Oxford, 1991, Chapter 1.5; c) M. E. Jung, in Comprehensive Organic Synthesis, Vol. 4 (Eds.: B. M. Trost, I. Fleming), Pergamon, Oxford, 1991, Chapter 1; d) L. Kurti, B. Czakó, in Strategic Applications of Named Reactions in Organic Synthesis, Elsevier, Amsterdam, 2005; e) J. J. Li, in Name Reactions: A Collection of Detailed Mechanisms and Synthetic Applications. 5th ed., Springer, Berlin, 2014.

[2] An excellent review on organocopper-mediated regioselective 1,4-addition: a) N. Krause, A. Gerold, Angew. Chem. Int. Ed. Engl. 1997, 36, 186-204; Angew. Chem. 1997, 109, 194-213, and references therein. Selected classical examples of the preparation and application of "lower order" organocopper reagents in 1,4-addition: b) M. S. Kharasch, P. O. Tawney, J. Am. Chem. Soc. 1941, 63, 2308-2316; c) H. Gilman, R. G. Jones, L. Woods, J. Org. Chem. 1952, 17, 1630-1634; d) G. H. Posner, Org. React. 1972, 19, 1-113; e) G. H. Posner, in An Introduction to Synthesis Using Organocopper Reagents. Wiley, New York, 1980. Selected reviews on the preparation and application of "higher order" organocopper reagents in 1,4-addition: f) B. H. Lipshutz, R. S. Wilhelm, J. A. Kozlowski, Tetrahedron 1984, 40, 5005-5038; g) B. H. Lipshutz, Synthesis 1987, 325-341; h) B. H. Lipshutz, S. Sengupta, Org. React. 1992, 41, 135-631. Selected reviews on copper-catalyzed or -mediated enantioselective conjugate addition reactions: i) A. Alexakis, J. E. Bäckvall, N. Krause, O. Pàmies, M. Diéguez, Chem. Rev. 2008, 108, 2796-2823; j) B. E. Rossiter, N. M. Swingle, Chem. Rev. 1992, 92, 771-806.

[3] An excellent review on rhodium-catalyzed enantioselective conjugate addition reactions: a) T. Hayashi, K. Yamasaki, Chem. Rev. 2003, 103, 2829-2844. The first enantioselective conjugate addition catalyzed by a chiral rhodium complex: b) M. Sawamura, H. Hamashima, Y. Ito, J. Am. Chem. Soc. 1992, 114, $8295-8296$.

[4] Selected reviews on enantioselective conjugate additions catalyzed by other soft metals: a) M. P. Sibi, S. Manyem, Tetrahedron 2000, 56, 8033-8061; b) N. Krause, A. Hoffmann-Röder, Synthesis 2001, 0171-0196; c) J. Christoffers, G. Koripelly, A. Rosiak, M. Rössle, Synthesis 2007, 1279-1300.

[5] An excellent review on different types of carbon nucleophiles for conjugate additions: A. G. Csákÿ, G. D. L. Herrán, M. C. Murcia, Chem. Soc. Rev. 2010, 39, 4080-4102.

[6] a) C. Bruneau, P. H. Dixneuf, in Ruthenium Catalysts and Fine Chemistry, Springer, Berlin, 2004. An early example on ruthenium(II)-catalyzed Michael addition through $\mathrm{C}-\mathrm{H}$ activation: b) S.-I. Murahashi, T. Naota, H. Taki, M. Mizuno, H. Takaya, S. Komiya, Y. Mizuno, N. Oyasato, M. Hiraoka, M. Hirano, A. Fukuoka, J. Am. Chem. Soc. 1995, 117, 12436-12451.

[7] Selected conceptual papers on umpolung chemistry: a) R. Breslow, J. Am. Chem. Soc. 1958, 80, 3719-3726; b) D. Seebach, Angew. Chem. Int. Ed. Engl. 1979, 18, 239-258; Angew. Chem. 1979, 91, 259-278. Recent examples of umpolung of imine reactivity: c) R. Brehme, D. Enders, R. Fernandez, J. M. 
Lassaletta, Eur. J. Org. Chem. 2007, 5629-5660; d) Y. Wu, L. Hu, Z. Li, L. Deng, Nature 2015, 523, 445-450; e) J. E. Baldwin, R. Aldlington, J. C. Bottaro, J. N. Kolhe, I. M. Newington, M. W. D. Perry, Tetrahedron, 1986, 42, 4235.

[8] a) X.-J. Dai, C.-J. Li, J. Am. Chem. Soc. 2016, 138, 5433-5440. b) Early Ir-catalyzed deoxygenation chemistry: J.-L. Huang, X.J. Dai, C.-J. Li, Eur. J. Org. Chem. 2013, 6496-6500.

[9] a) The carbonyl addition reaction: H. Wang, X.-J. Dai, C.-J. Li, Nat. Chem. 2016, AOP paper, DOI: 10.1038/NCHEM.2677.b) The imine addition reaction: N. Chen, X.-J. Dai, H. Wang, C.-J. Li, Angew. Chem. Int. Ed. 2017, DOI: 10.1002/anie.201610578; Angew. Chem. 2017, DOI: 10.1002/ange.201610578.

[10] a) A. D. Garnovskii, B. I. Kharissov, in Synthetic Coordination and Organometallic Chemistry, CRC Press, Boca Raton, FL, 2003; b) M. J. Clarke, in Metals Ions in Biological System, Dekker, New York, 1980.

[11] Despite the unclear role of CsF in the current reaction, it has been known to favor transmetallation in cross-coupling reactions such as the Suzuki and the Stille couplings: a) S. W. Wright, D. L. Hageman, L. D. McClure, J. Org. Chem. 1994, 59, 6095-6097; b) A. F. Littke, G. C. Fu, Angew. Chem. Int. Ed. 1999, 38, 2411 2413; Angew. Chem. 1999, 111, 2568-2570. The fluoride anion is also known to enhance nucleophilicity as a strong hydrogenbond acceptor: c) J. H. Clark, J. Emsley, J. Chem. Soc. Dalton
Trans. 1975, 2129 -2134; d) J. H. Clark, D. G. Cork, H. W. Gibbs, J. Chem. Soc. Perkin Trans. 1 1983, 2253-2258.

[12] Phosphines were previously reported to catalyze Michael additions alone: a) C. Gimbert, M. Lumbierres, C. Marchi, M. Moreno-Mañas, R. M. Sebastián, A. Vallribera, Tetrahedron 2005, 61, 8598-8605; b) C. Gimbert, M. Moreno-Mañas, E. Pérez, A. Vallribera, Tetrahedron 2007, 63, 8305-8310.

[13] W. H. Miles, E. A. Dethoff, H. H. Tuson, G. Ulas, J. Org. Chem. 2005, 70, 2862-2865. Note: pre-synthesized 2-furylhydrazone was cautiously distilled prior to use in this reaction.

[14] Examples of strong chelation of pyridine and its analogues to late transition metal catalysts: a) E. S. Raper, Coord. Chem. Rev. 1996, 153, 199-255; b) D. A. Colby, A. S. Tsai, R. G. Bergman, J. A. Ellman, Acc. Chem. Res. 2012, 45, 814-825.

[15] Examples of highly functionalized organometallic reagents: a) P. Knochel, W. Dohle, N. Gommermann, F. F. Kneisel, F. Kopp, T. Korn, I. Sapountzis, V. A. Vu, Angew. Chem. Int. Ed. 2003, 42, 4302-4320; Angew. Chem. 2003, 115, 4438-4456; b) A. Krasovskiy, P. A. Knochel, Angew. Chem. Int. Ed. 2004, 43, $3333-$ 3336; Angew. Chem. 2004, 116, 3396-3399.

Manuscript received: January 3, 2017

Revised: January 16, 2017

Final Article published: February 16, 2017 